\title{
Le déclin de l'emploi est-il celui du salariat? Vers un modèle de la qualification personnelle
}

Does Employment decline also mean Salary's one? Towards a model of personal qualification

\section{Bernard Friot}

\section{OpenEdition}

\section{Journals}

Édition électronique

URL : http://journals.openedition.org/travailemploi/5167

DOI : 10.4000/travailemploi.5167

ISSN : 1775-416X

\section{Éditeur}

DARES - Ministère du Travail

\section{Édition imprimée}

Date de publication : 15 juin 2011

Pagination : 61-70

ISSN : 0224-4365

\section{Référence électronique}

Bernard Friot, " Le déclin de l'emploi est-il celui du salariat ? Vers un modèle de la qualification personnelle», Travail et Emploi [En ligne], 126 | avril-juin 2011, mis en ligne le 29 février 2012, consulté le 10 décembre 2020. URL : http://journals.openedition.org/travailemploi/5167 ; DOI : https://doi.org/ 10.4000/travailemploi.5167 


\title{
Le déclin de l'emploi est-il celui du salariat? Vers un modèle de la qualification personnelle
}

\author{
Bernard Friot $\left(^{*}\right)$
}

\begin{abstract}
Travail et emploi a consacré son numéro 125 à des contributions à dominante empirique sélectionnées parmi les interventions présentées lors des XXIX ${ }^{e s}$ Journées de l'Association d'économie sociale. Le thème d'étude de ces journées était le lien existant entre " emploi et politiques sociales»; les débats $y$ ont porté notamment sur l'avenir de la protection sociale et sur "l'inversion progressive du statut de la protection sociale» en France. Dans ce numéro, nous avons retenu une contribution également présentée lors de ces journées, mais qui est plus prospective. L'auteur y développe une lecture des transformations de l'emploi, du marché du travail et du salariat dans les économies capitalistes depuis la fin de la Seconde Guerre mondiale. Sur l'exemple de la France et avec quelques références à l'Union européenne, il décrit comment les réformes en cours ont pour lui la finalité de réduire les droits salariaux affectés au poste de travail et de vider l'emploi de son contenu salarial. Il propose ensuite un projet d'organisation sociale alternative, fondée sur le salariat universel et la qualification personnelle qui, comme utopie, constitue un horizon de réflexion à même de stimuler un débat sur l'avenir de nos économies (chapeau de la rédaction).
\end{abstract}

L'emploi, tel qu'il se construit dans les années 1950-1960, est aujourd'hui en cause. L'invocation de l'employabilité le transforme : d'institution de qualification du poste de travail et de sécurisation des rapports de travail, support de nombreux droits sociaux, l'emploi devient une asymptote que ne rejoint jamais réellement la trajectoire du salarié. Celui-ci est en permanence soupçonné d'une «distance à l'emploi», et cette employabilité qu'il va certes être incité à améliorer et aidé pour ce faire, le disqualifie, au sens propre, en permanence.

Ce déclin de l'emploi comme support des droits salariaux signifie-t-il celui du salariat? C'est une opinion courante, fondée sur le fait que le droit du travail et de la sécurité sociale s'est construit sur la reconnaissance de la subordination du salarié à un employeur, construction dont l'emploi est, dans les années 1970, l'expression aboutie. Elle a été mise en forme par l'ouvrage désormais classique de Robert CAstel (1995) : le salariat a connu son heure de gloire pendant les Trente Glorieuses, quand l'État social doublait la propriété privée d'une propriété sociale pour des salariés qui, fordisme oblige, bénéficiaient ainsi d'institutions macro sociales venant rendre effectif l'échange de la sécurité contre la subordination. La vague néo-libérale est venue mettre en crise le salariat, ce qui conduit l'auteur à retenir le concept de précariat.

(*) IDHE, université Paris Ouest Nanterre, chercheur invité à la MSH Lorraine; bfriot@u-paris10.fr

\section{Marché du travail, emploi et salaire}

Ce large consensus sur le déclin du salariat mérite d'être interrogé. Encore faut-il poser le salariat dans toute son épaisseur conceptuelle et non pas le réduire à l'addition des salariés. Le déni de la théorisation du salariat trouve son illustration dans l'acceptation par les chercheurs continentaux de la typologie d'Esping-Andersen qui, loin de valoriser le modèle continental, précisément inventeur des institutions salariales, le renvoie aux ténèbres pré-marchandes du corporatisme conservateur ${ }^{(1)}$. $\mathrm{Si}$ on définit le salariat, non pas comme l'ensemble de ceux qui ont un statut juridique de salariés, mais comme l'ensemble articulé des institutions salariales, son cœur est le salaire qui, loin de se réduire à la relation d'emploi, peut la subvertir. Les institutions salariales sont décisives dans la dynamique économique et sociale contemporaine, qu'il s'agisse du salaire à la qualification, des conventions collectives et statuts; des cotisations sociales et de la sécurité sociale : pensions, couverture des soins de santé, allocations familiales, indemnisation du chômage; du droit du travail, de l'ordre public social, du droit de grève, de la négociation collective, des juridictions du travail; des syndicats, de la démocratie sociale, de la représentation des salariés dans les entreprises; de la fonction publique et des

(1) Voir la critique dévastatrice que fait Robert SALAIs (2005) de Gøsta Esping-Andersen (1990), The three worlds of welfare capitalism, Oxford, Polity Press. 
services publics de l'État social. Toutes ces institutions salariales sont depuis les années 1980 l'objet de déconstructions que leurs promoteurs appellent des «réformes», et nous adopterons ce terme indigène même si le sens courant associait jusqu'alors à ce mot l'idée d'un changement dans le progrès. De même, nous appellerons «réformateurs» ceux qui dans le champ gouvernemental et de l'expertise, soit sont à l'initiative de ces réformes demandées par le patronat, soit en construisent l'argumentaire (CAstel, 2009). Ces réformes s'inscrivent toutes dans la même dynamique, que l'objet de ce texte est d'analyser. Mettre en évidence a posteriori leur cohérence ne signifie pas, évidemment, qu'elles sont la mise en œuvre d'un projet. Les réformateurs ne sont pas des comploteurs, et les réformes sont une construction sociale pragmatique dont on ne peut pas donner à l'avance l'issue : au contraire, nous pensons qu'elles peuvent conduire à des issues contradictoires.

Les réformes mettent les institutions salariales «au service de l'emploi»: c'est l'enjeu essentiel de la Stratégie européenne pour l'emploi. Elles changent ainsi le sens de l'emploi, qui n'est plus la matrice des droits salariaux mais l'occasion de leur mise en cause au nom de la «défense de l'emploi». C'est que ces droits salariaux, loin d'être fonctionnels au capital, le fragilisent : la cotisation sociale, par exemple, rend inutiles la prévoyance et l'accumulation du capital pour assumer des engagements massifs et de long terme; une prestation sociale comme la pension attribue jusqu'à leur mort une part de leur salaire à des salariés débarrassés de la subordination et qui trouvent dans cette situation de salaire à vie un grand bonheur à travailler. On voit à travers la brève évocation de ces exemples combien les institutions salariales peuvent être subversives de celles du capital, à savoir :

- la propriété lucrative, avec son cortège d'investisseurs et d'actionnaires et l'éloge de la prévoyance : la propriété de titres financiers donne droit à une partie de la monnaie correspondant à la valeur actuelle du travail d'autrui, que ce soit aujourd'hui (dans le dividende ou le taux d'intérêt) ou demain lors de la liquidation des titres;

- le marché du travail, qui repose sur la propriété lucrative : des «employeurs» exproprient des «employés» de leur capacité créatrice, transformée en force de travail. Ce déni de la qualification de producteurs ramenés au statut d'êtres de besoin à la recherche d'un revenu et se subordonnant à des employeurs les réduit à être des demandeurs d'emploi. Les seuls producteurs sont ainsi contraints de prouver leur employabilité tout en perdant la maîtrise de la définition des fins et des moyens de leur travail (subordination capitaliste);

- la marchandise, seule forme légitime des produits du travail, car le fait que les biens et services sont produits pour être vendus est une autre condition de l'extraction de la survaleur par les actionnaires. Le non-marchand n'est acceptable que pour autant qu'il est nécessaire à l'affirmation de la marchandise et de la propriété lucrative, comme dans le cas de la pension publique, condition d'une épargne retraite sur les marchés financiers. Le règne de la marchandise est assuré par le fait que la marchandise capitaliste est la seule médiation de la création monétaire lors du crédit bancaire aux entreprises.

Les réformes tentent d'éviter la marginalisation de ces fondements du capital par les institutions salariales en changeant le sens de l'institution jusque-là porteuse de ces dernières, l'emploi. Mais ce support de l'emploi était très ambigu, puisqu'il pérennisait le statut de salariés définis par la subordination. Toute la question du devenir des réformes est de savoir si la mise en cause de l'emploi ne sera pas l'occasion, au contraire, de l'invention d'un nouveau support des droits salariaux qui, n'étant plus portés par l'emploi, seront émancipés de la subordination et du marché du travail. Or l'examen attentif des mutations et des débats actuels laisse entrevoir dans la qualification personnelle une telle institution substitut possible de l'emploi et porteuse d'un salariat subversif du capitalisme. Par qualification personnelle, nous entendons le fait que, dans le grade de la fonction publique, dans le diplôme protégé de la profession libérale, et dans le salaire à vie des retraités, la qualification (et donc le salaire) est l'attribut de la personne elle-même, et non pas du poste de travail comme dans l'emploi. La qualification ne doit pas être confondue avec la certification. Certes le diplôme est attaché à la personne, mais tant qu'il n'est pas soit protégé, soit actualisé dans un emploi ou un grade, il ne vaut aucun salaire. Qualifier, c'est attacher un salaire, à un poste de travail (dans le cas de l'emploi) ou à une personne (grade, pension comme salaire à vie). L'école certifie, elle ne qualifie pas. Comment la qualification personnelle s'inscrit-elle dans la trilogie classique «qualification de la personne, de l'emploi et du travail»? Elle ne se confond pas avec la certification de la personne, contrairement à l'usage courant de la «qualification de la personne» qui désigne en réalité son niveau de diplôme. Elle s'oppose à la qualification du poste de travail (désignée comme «qualification de l'emploi»). Elle s'affranchit de la mesure de la qualification du travail, car elle attribue à son titulaire un niveau de participation potentielle à la production, qu'elle ne mesure pas dans son travail hic et nunc.

Ces puissances du salariat (FrIOT, 2012) se jouent dans une dynamique que nous proposons d'analyser par l'articulation conflictuelle des idéaux-types du salarié, de l'employé et du travailleur. Nous chargeons bien sûr ces idéaux-types d'un sens théorique, et il ne faut pas les prendre dans leur acception courante : nous désignons comme «employé» le travailleur défini comme titulaire 
d'un emploi, comme «salarié» celui qui est défini par son salaire, et réservons le terme habituellement générique de «travailleur» au travailleur qui se définit sur le marché du travail. Et c'est toute la distance que prennent les constructions conjointes du marché du travail, de l'emploi et du salaire qui fait le conflit entre ces trois figures. La dynamique des dernières décennies peut ainsi être interprétée comme l'articulation conflictuelle de trois logiques, toutes trois inégalement présentes aujourd'hui dans le débat public. La logique de l'emploi telle qu'elle s'est construite entre 1945 et le début des années 1980 était déjà porteuse d'une logique salariale qui la dépassait : ce sera l'objet de la première partie. Et les réformes, qui promeuvent l'employabilité et le marché du travail contre la montée en puissance des institutions salariales, offrent à ces dernières des possibilités porteuses non d'un déclin, mais aussi, dans une utopie encore à construire, d'un progrès du salariat : ce sera l'objet des deuxième et troisième parties. Ce faisant, on examinera les logiques de l'emploi, du marché du travail et du salaire (sans oublier qu'elles n'existent que dans leur relation) selon leur rapport aux institutions capitalistes de la propriété lucrative, du marché du travail et de la création monétaire par la médiation de la marchandise.

\section{La logique de l'emploi jusqu'aux années 1980}

La logique qui prévaut autour de 1968 est celle d'institutions qui viennent doubler la propriété lucrative par la cotisation sociale, le marché du travail par la grille des salaires des conventions collectives, la marchandise par le service public, la création monétaire des banques commerciales par le financement des dépenses publiques par la banque centrale. Dans cette distinction entre emploi et hors emploi, marchand et non marchand, marché du travail et statut, un espace pré-salarial se construit contre la logique capitaliste, y compris lorsque ses institutions sont mises en place sur initiative patronale, comme l'ont été au départ les cotisations ou de nombreux dispositifs conventionnels.

Inspiré de la fonction publique quoiqu'en différant fondamentalement, l'emploi dans le secteur privé est à la fois une construction patronale et une conquête des travailleurs qui les libère partiellement du fardeau du marché du travail. Comme dans la fonction publique, la dimension corporatiste est essentielle dans une évolution liée à la fermeture de la branche (banque) ou de l'entreprise, qu'elle soit paternaliste (sidérurgie) ou à statut (SNCF, EDF). Ce sont dans ces lieux échappant en interne à la logique du marché du travail que la figure de l'employé a pu se construire pour le personnel permanent, en symétrique de celle de l'employeur, par négociation collective d'un statut ou d'accords de branche et d'entreprise. Statuts et dispositifs conventionnels dressent des hiérarchies de qualification, le plus souvent liées aux métiers, et y font correspondre des grilles de salaire respectées par les employeurs. Des régimes complémentaires au même périmètre bien circonscrit, financés directement par les employeurs avec ou sans cotisation sociale, avec épargne dans l'entre-deux-guerres et sans épargne à compter des années 1940, reconnaissent des temps dits «horsemploi» (maladie, invalidité, retraite) parce que fortement corrélés à l'emploi.

La dynamique de l'emploi marginalise ainsi le marché du travail en créant des marchés internes ${ }^{(2)}$ de grandes entreprises ou de branche, avec à l'intérieur de ces marchés internes une régulation par conventions collectives ou par statuts édictant des tarifs, des règles de mobilité, de conditions de travail, des règles de l'embauche et du licenciement. Cela ne vaut pas pour tous les secteurs, que l'on pense à l'hôtellerie, à la réparation automobile, au commerce. Mais dans de nombreuses branches ou grandes entreprises on assiste à la construction conjointe, dans le conflit et la négociation collectifs, des organisations syndicales représentant des titulaires d'emplois et des organisations de patrons qui deviennent des employeurs au sens où ils s'inscrivent eux aussi dans une logique d'affectation au poste de travail d'un certain nombre de droits salariaux, c'est-à-dire de transformation de postes de travail en emplois. Cela dit, la logique fondamentale du marché du travail est maintenue, puisque la qualification et le salaire, les droits de protection contre le licenciement ou les règles de l'organisation du travail sont un attribut du poste de travail et non pas de la personne des employés. Même si les marchés internes ou professionnels garantissent au titulaire d'un contrat de travail une carrière dans l'entreprise ou dans sa profession, ce qui le préserve de l'obligation de retour sur le marché du travail entre deux emplois, c'est le poste qu'il occupe qui est payé - parce que c'est le poste qui est qualifié, pas lui. Aucun examen professionnel ne lui attribue une qualification et donc un salaire à vie, même si, dans la pratique, son contrat de travail peut être le support d'une longue carrière ascendante.

À côté de l'emploi en train de se construire existe une forme plus ancienne au demeurant que lui, et qui, elle, ignore le marché de travail : la fonction publique. Le principe fondateur de la fonction publique est que les droits salariaux sont liés au grade et non pas au poste, au grade c'est-à-dire à la qualification personnelle de l'individu tel qu'elle lui est reconnue à travers un concours ou un examen professionnel. Aussi les fonctionnaires échappentils à une logique de marché du travail puisque ce

(2) En Allemagne, une évolution parallèle s'opère dans une logique de marchés professionnels plutôt que de marchés internes. 
n'est pas leur emploi qui détermine leurs droits. Cette exception n'est pas sans importance, d'abord numérique, et puis parce qu'elle va inspirer les statuts des entreprises publiques et constituer un modèle pour les négociateurs des conventions collectives dans le secteur privé. Mais demeure toujours entre la fonction publique et le secteur privé la grande différence d'une qualification liée à la personne, alors que dans le secteur privé, la qualification (et tous les droits qui vont avec) est liée à l'emploi.

$\mathrm{Du}$ point de vue de l'émancipation du travail des institutions capitalistes, on peut donc, tout en insistant sur son aspect limité et corporatiste, souligner le caractère positif de l'emploi dans cette période des années 1950-1970. Il s'affirme comme l'attachement au poste de travail (et aux postes de travail successifs dans le cadre d'un contrat de travail) de droits qui font des travailleurs des «employés», des employés en négociation collective avec des «employeurs", l'employeur retenant dans son ombre l'actionnaire. Caractère d'autant plus positif que cette logique de l'emploi va être la matrice du salaire. Du salaire direct, nous l'avons $\mathrm{vu}$ : la convention collective définit les salaires selon une grille qui s'impose à l'employeur. Mais aussi de l'autre dimension du salaire, davantage contestatrice encore des institutions du capital, à savoir la cotisation sociale qui connait alors une montée en puissance tout à fait considérable comme réponse patronale à la revendication de hausse des salaires directs, comme nous l'avons montré dans Puissances du salariat (FRIOT, 1998, 2012). Témoignage des potentialités subversives du capitalisme d'initiatives patronales au départ, la cotisation sociale est une mutualisation du salaire qui déconnecte ce dernier de l'emploi. Avec ou sans emploi, il y a droit à l'assurance-maladie, aux allocations familiales, à la retraite, au chômage : le salaire a une application beaucoup plus large que le seul champ de l'emploi et cette distinction entre le salaire et l'emploi est un élément supplémentaire de marginalisation du marché du travail. Mais plus encore la cotisation sociale marginalise la propriété lucrative. En matière de retraites, la cotisation sociale opère la démonstration qu'un engagement aussi massif (13\% du PIB aujourd'hui) et de long terme (des dizaines d'années) que les pensions peut être honoré sans aucune épargne, sans aucun taux d'intérêt, sans accumulation financière, sans crédit, bref sans toutes les institutions qui gravitent autour de la propriété lucrative. La cotisation sociale, en tant qu'affectation au salaire d'une partie du flux de la valeur qui va être immédiatement transformée en prestations sociales, peut être vue comme une technologie anticapitaliste qui montre l'inutilité de la propriété lucrative. Enfin, troisième institution du capital, la marchandise, et troisième contestation opérée par le salaire tel qu'il se construit dans la matrice de l'emploi : le financement du non-marchand. Ainsi la cotisation sociale, la partie du salaire mutualisée entre les employeurs, finance la production nonmarchande de soins dans l'assurance-maladie.

Ce salaire socialisé l'est selon des logiques qui sont partiellement restées corporatistes et la volonté patronale d'émiettement de la sécurité sociale selon les risques et les régimes trouve des accroches du côté syndical, et surtout là où les marchés internes sont les plus construits et la logique de l'emploi la plus élaborée : le refus des électriciens ou des cheminots de la CGT d'entrer dans le régime général, la négociation par FO ou la CFTC (on est avant 1964 et la transformation de cette dernière en CFDT) de régimes complémentaires vont fragiliser une institution que boudent les fonctionnaires (certes rattachés au régime général pour la santé, mais formellement, puisque leurs mutuelles ont une concession de gestion à travers laquelle la mutualité conservera une place qui, après 1985 , se révélera le cheval de Troie de la réforme de l'assurancemaladie). La logique du salaire est à la fois portée et fragilisée par celle de l'emploi et par le cantonnement de la fonction publique.

Examinons enfin ce qu'il en est de la création monétaire. Sa forme dominante passe par le crédit aux entreprises des banques commerciales qui créent de la monnaie par anticipation de la valeur attribuée aux marchandises capitalistes. Mais avec le circuit du trésor, avec la banque centrale une création monétaire a pour médiation la dépense publique. On retrouve le partage des tâches caractéristique de la logique de l'emploi : création monétaire dans l'institution capitaliste qu'est le prêt bancaire aux entreprises, mais aussi création monétaire pour financer les services publics. Tout comme le travail subordonné dans l'emploi est doublé par la reconnaissance salariale d'un hors emploi et donc du travail non subordonné, le marchand l'est par le non-marchand.

Parce qu'elle ouvre ainsi un espace à des institutions contestatrices des institutions du capital, la dynamique salariale de l'emploi, comme nous allons le voir dans la seconde partie, a été mise en cause par les réformateurs dès qu'elle s'est affirmée au lendemain de mai 1968. Comme l'a montré Corinne GoBIN (2005), à compter du milieu des années 1970, une contre-offensive de la classe dirigeante, largement orchestrée au niveau européen surtout à partir de l'Acte unique de 1985 et de la construction du marché intérieur, va mener les réformes au nom de la libre circulation des capitaux, des marchandises et des personnes, de la compétitivité des entreprises et de la modernisation du marché du travail. Précisons que, de même que la construction de l'emploi n'avait pas effacé le marché du travail, de même mettre en avant le marché du travail comme le font les réformes ne remet pas en cause l'hétéronomie du travailleur tel qu'elle s'exprime dans la logique de l'emploi. Le marché du travail devient l'élément central relativement auquel peut se déployer une 
logique de l'emploi : nous sommes dans un mouvement inverse de celui de la période précédente, qui affirmait l'emploi en marginalisant le marché du travail. Les réformes s'inscrivent dans la proposition communautaire de flexicurité qui réassume la logique de l'emploi selon des modes plus adéquats à un capitalisme financiarisé.

Mais, en contradiction avec cette logique réformatrice, la dynamique du salaire à vie à la qualification personnelle peut conduire à supprimer le marché travail et les emplois : ce sera l'objet de la troisième partie. Cette dynamique, traditionnellement celle des fonctionnaires et qui s'affirme parmi les retraités dont la pension se rapproche de leur salaire (au début des années 1990, le taux de remplacement $\mathrm{du}$ dernier salaire net par la première pension nette atteint $84 \%$ pour les salariés à carrière complète nés en 1930, avant de régresser au fil des différentes réformes), est portée en France par des revendications syndicales comme celle d'un «nouveau statut du travail salarié» à la CGT. Elle s'appuie sur les droits salariaux conquis dans la dynamique de l'emploi et sur le précédent de la fonction publique, mais en dépassant leur corporatisme, ce qui la rend porteuse d'un «salariat» riche d'institutions aptes à remplacer les institutions du capital : le marché du travail par la qualification personnelle, le droit de propriété lucrative par l'investissement salarial, la médiation de la marchandise par celle de la qualification pour la création monétaire.

\section{Les réformes depuis les années 1980 : le marché du travail contre le salaire}

La dynamique du marché du travail a pour objectif de restaurer comme centrales et même comme exclusives les institutions du capital alors que la logique de l'emploi les avait doublées d'institutions potentiellement ou déjà anticapitalistes. La première manifestation de cette réforme est la réaction monétariste qui à compter de la seconde moitié des années 1970 interdit le financement de la dépense publique par la banque centrale : le circuit du trésor est démantelé et ne reste légitime que la création monétaire par anticipation du prix des marchandises dans le prêt bancaire. Cette impossibilité de la création monétaire pour financer des dépenses publiques s'accompagne d'une délégitimation de ces dernières et d'une remise en cause de la fiscalité qui tendent à imposer la production marchande, ou à ne tolérer la production non marchande que pour autant qu'elle est au service de la production marchande. Il reste bien un espace pour un secteur public, mais il doit être réformé pour servir la logique de l'accumulation financière. Que l'on songe par exemple à la pension publique qui, dans les pays où elle était peu importante comme au Royaume-Uni, va voir sa part augmenter parce que l'accès à l'épargne retraite sous les formes vraiment marchandes de portefeuilles de titres (individuels ou professionnels) suppose qu'une pension publique assure aux travailleurs épargnants un horizon et une sécurité certes insuffisante (ce qui nécessite l'épargne), mais suffisante pour que l'épargne ne s'enferme pas dans les niches de l'immobilier et aille dans le grand vent des marchés des capitaux ${ }^{(3)}$.

En même temps, la financiarisation du capital pose l'actionnaire, la propriété lucrative, le crédit, «l'investisseur» en produits financiers comme des figures plus légitimes que l'employeur et les employés : les nouvelles formes de management et les nouveaux indicateurs de prises de décisions économiques sont au service de la production de valeur pour l'actionnaire, au service de la sécurité de taux de retours bien supérieurs aux gains de productivité, tandis que l'insécurité est reportée sur les salariés ou sur l'activité de production dans l'économie réelle. La bulle financière va progressivement se constituer sur la base d'une modération salariale dont le principal aiguillon est le chantage à l'emploi, la pratique de la délocalisation, la mise en cause des conventions collectives par la soustraitance. Le périmètre ainsi mouvant de l'entreprise l'est rendu encore davantage au gré des OPA, dans la mesure où la production de valeur pour l'actionnaire fait que les stratégies des dirigeants peuvent être déterminées par des indicateurs boursiers. Dans cette nouvelle configuration de l'entreprise, l'employeur s'efface derrière l'actionnaire, la direction des ressources humaines conquiert une place fondamentale détachée des logiques productives, et l'institution capitaliste de l'accumulation financière et du droit de propriété lucrative retrouve tout son lustre. Pour cela, il faut bien sûr que la cotisation sociale soit stigmatisée, contestée, récusée, désignée comme taxe sur le travail par exemple, remplacée par des dotations budgétaires aux caisses de sécurité sociale, ce qui va rendre encore plus difficile la dépense publique et donc réduire l'espace de la fonction publique et du non-marchand.

S'agissant du marché du travail, les réformes s'emploient à le globaliser en en finissant avec les marchés internes. Dans la mesure où ils ont été le vecteur de la logique de l'emploi, c'est donc l'emploi qui est en cause. Non pas en tant qu'il est consubstantiellement lié à l'existence d'un marché du travail, mais en tant qu'il est porteur de droits salariaux. Dénoncés comme inégalitaires et sources de discrimination entre inclus et exclus, ceux-ci sont remis en cause à travers la promotion de «l'employabilité» à la place de la qualification liée

(3) Implementing an integrated package of pension reforms : The Final Report of the Pensions Commission, 4 avril 2006 [http://www.pensionscommission.org.uk/publications/2006/ final-report/final_report.pdf]; voir aussi LE LANN (2006). 
au poste de travail. Ce qui est mis en avant, ce n'est plus d'abord le poste de travail, sa qualification et la sécurisation des droits liés, ce n'est donc plus la qualité et la sécurité de l'emploi, c'est la sécurité de la mobilité à travers une «sécurisation des parcours professionnels », pour reprendre la thématique dominante aujourd'hui en France ${ }^{(4)}$. Cette version française de la flexicurité communautaire repose sur l'employabilité, c'est-à-dire sur le fait que le travailleur est toujours suspecté d'entretenir une distance à l'emploi qu'il est dans l'injonction permanente de réduire. On voit donc bien que le mot «emploi»a changé radicalement de sens : non plus une protection du travailleur liée à une qualification du poste de travail, mais le terme jamais atteint de son employabilité, l'instrument donc de sa permanente disqualification.

Ce déplacement se traduit chez les réformateurs (cf. par exemple Cahuc, KRAmarz, 2004) par l'affirmation de la nécessité d'une médiation efficace sur le marché du travail. C'est toute la thématique du service public de l'emploi (SPE), un SPE ayant une obligation de résultat, et donc mieux financé grâce à une taxe de type pollueur-payeur sur les entreprises qui licencient. Le SPE profile les chômeurs, c'est-à-dire mesure leur distance à l'emploi, puis les affecte à des agences de reclassement dont l'obligation de résultat est matérialisée en particulier dans leur mode de financement, en fonction du placement effectif des personnes qui leur ont été confiées. Cette obligation de résultat étant toujours accompagnée symétriquement d'une obligation pour les salariés d'accepter les emplois «convenables», potentiellement moins qualifiés que leur emploi perdu. Ce caractère central du SPE s'accompagne d'un droit à la formation tout au long de la vie, et ce droit, ainsi que les autres droits garants de l'amélioration de l'employabilité, ne doivent plus être liés au poste de travail mais à la personne. Le droit au reclassement, l'accès aux prestations du SPE, le droit à la formation ne doivent plus être liés au type de poste de travail, à la taille ou au secteur de l'entreprise, au type de contrat de travail, mais ils doivent être universels et transférables d'une entreprise à l'autre avec obligation pour le nouvel employeur de les honorer au niveau où ils ont été acquis précédemment.

Acquis comment? Là encore, nous sommes dans une logique tout autre que la logique salariale antérieure, parce que la marginalisation de l'emploi au bénéfice du marché du travail est l'occasion d'une marginalisation massive du salaire. Il est d'ailleurs significatif que le terme «salaire» soit exclu du débat public et remplacé par celui de «pouvoir d'achat». D'une part, dans la mesure où la qualification du poste, sa sécurité, sont remises en cause

(4) Dont témoigne l'accord de janvier 2008 sur la «modernisation du marché du travail». (au bénéfice de l'employabilité et de la sécurisation des mobilités), il en est de même pour le salaire à la qualification, le respect de la grille salariale, la négociation collective de branche, l'ordre public social, qui font que le contrat de travail doit respecter toute une hiérarchie des normes. Avec d'ailleurs une forte impulsion communautaire dont témoignent encore les arrêts récents de la Cour de justice européenne ${ }^{(5)}$ qui affirment le principe du droit du travail du pays d'origine sous réserve du respect du seul droit national minimal du pays d'accueil, ce qui y détruit toute l'épaisseur de l'ordre public social.

D'autre part, le salaire socialisé est largement mis en cause, et en particulier la cotisation sociale dont nous avons vu toute la portée anticapitaliste. $\mathrm{La}$ cotisation qui générait une prestation en répartition fonction du salaire est remplacée soit par l'impôt de la CSG, soit par un dispositif de piliers, un premier pilier de revenu différé et garanti par l'État sur le modèle de la pension publique suédoise ${ }^{(6)}$ et un second pilier d'épargne salariale. Le salaire a disparu, nous sommes dans la logique d'une prévoyance qui accumule sur des comptes individuels les droits à pensions tout comme s'accumulent sur des comptes individuels des droits à formation, à RTT, à une couverture santé complémentaire, au chômage : tout peut devenir l'objet d'une épargne notionnelle qui ne génère pas d'accumulation financière mais qui, fonctionnant selon la logique de l'épargne, remplace le salaire et redonne leur légitimité à une propriété lucrative et à une accumulation financière que la cotisation sociale avait fortement contestées (Le LanN, 2009).

Nous n'avons donc plus affaire à des employés, mais à des travailleurs prévoyants : l'employabilité remplace la qualification, la garantie d'une prévoyance liée au travail subordonné à travers des comptes épargne remplace le salaire de la cotisation sociale : le salaire socialisé cède la place au revenu différé (FRIOT, 2010) ou à l'accumulation financière en même temps que le binôme contrat de travail/ contrôle judiciaire remplace l'ordre public social. Tandis que seul le travail subordonné génère des droits, ceux-ci prennent des formes congruentes au marché du travail et à la propriété lucrative. Si on ajoute à ces éléments le retour aux marchandises comme seule médiation de la création monétaire et la prégnance des marchés financiers évoqués plus haut, on voit que, contre les prémices d'institutions salariales dont était porteuse la dynamique de l'emploi, ce sont les institutions du capital qui sont remises en selle.

(5) Arrêts Viking (CJCE 11/12/2007), Laval (18/12/2007), Ruffert (02/04/2008), contre le Luxembourg (19/06/2008).

(6) Modèle qu'Antoine Bozio et Thomas Piketty (2008) veulent implanter en France. 


\section{Un nouveau modèle en germe? De l'emploi à la qualification personnelle}

En rester là serait toutefois ne pas voir le caractère potentiellement contradictoire du mouvement dans lequel s'inscrit la logique réformatrice. La dynamique du marché du travail est en effet antinomique d'une autre dynamique, celle qui va du salaire à la qualification personnelle dont on présente ici les grands traits structurels, toujours relativement aux institutions du capital que sont le marché du travail, la création monétaire à l'occasion des marchandises, la propriété lucrative. C'est la dynamique la plus récente parce qu'elle ne trouve une expression construite que depuis quelques années avec la revendication par la CGT d'un nouveau statut du travail salarié, qui se réfère à l'occasion aux travaux d'Alain Supiot (SupIot, 1999). Certes elle ne part pas de rien : la sécurité sociale, les conventions collectives, la fonction publique en sont des anticipations puissantes, aujourd'hui considérablement relayées par la croissance du poids des retraités payés par un salaire continué dans leur pension, un salaire à vie, donc.

Mais la dimension normative de ce que nous proposons ici est plus présente puisqu'il s'agit de réfléchir aux possibles qu'ouvre une dynamique qui part de la contestation de la dynamique de l'emploi non pas pour restaurer les institutions du capital, comme dans la dynamique du marché du travail, mais pour les remplacer par les institutions salariales et affirmer ainsi le salariat (FRIOT, 2007, 2009).

Au cœur de la logique du salaire à vie attaché à la qualification personnelle il y a, comme dans la précédente, le déplacement de droits liés à l'emploi vers la personne même des salariés. Cette dynamique s'inscrit dans l'actuelle relativisation de la logique de l'emploi, mais pousse à sa disparition en tant que celui-ci repose sur la négation de la qualification des personnes. Le modèle de sécurisation des parcours professionnels que nous venons d'analyser ne transfère en aucun cas la qualification (et donc le salaire) à la personne, puisqu'il s'agit de conforter le marché du travail en passant de la qualification des postes à l'employabilité des travailleurs. Là au contraire, l'employabilité céderait la place à la qualification personnelle. Si la qualification devient un attribut personnel qui ne peut pas être mis en cause à la baisse à l'occasion d'un changement de poste de travail ou de sa suppression, le salaire, qui est lié à la qualification, ne peut pas lui non plus être remis en cause. Nous sommes dans la situation d'un salaire tout au long de la vie et donc d'un salaire pour tous ${ }^{(7)}$. Ce salaire de la fin du lycée à la mort signifie que la pension est la poursuite du salaire (et il n'y a aucune raison que la qualification s'arrête avec l'entrée en retraite, qui pourra signifier tout autre chose qu'aujourd'hui), qu'il y a continuation du salaire entre deux emplois, qu'un salaire est attribué avant le premier emploi.

La déconnexion du salaire et de l'emploi est, selon cette logique, beaucoup plus radicale qu'avec la sécurité sociale. Le salaire universel est un salaire, pas un revenu : lié à la qualification, à sa progression au cours de la vie grâce au soutien d'institutions dédiées à la qualification des personnes, et à son échelle hiérarchique (par exemple dans une hiérarchie de 1 à 4 comme le propose la CGT), il n'a rien à voir avec un revenu universel d'existence forfaitaire. C'est un salaire tout au long de la vie et non pas, faut-il le préciser, un emploi à vie : au contraire ce salaire à vie est la condition de la mobilité, d'une mobilité choisie alors qu'aujourd'hui s'expriment surtout des mobilités contraintes dans le secteur privé (RAmaux, 2006). La question posée est celle de faire du salaire un droit politique, c'est-à-dire un attribut personnel irréversible qui affirme que chacun (y compris les personnes handicapées de naissance) participe à la production de valeur économique ${ }^{(8)}$. Mesure de cette potentialité, la qualification personnelle n'est ni la mesure de besoins d'une force de travail, ni la mesure du travail ou de son produit. Le droit de vote reconnaît une capacité à participer à la vie politique mais il ne mesure pas son produit. C'est la même démarche politique que de reconnaître une qualification à toute personne et d'instituer ainsi une majorité économique. À 16 ou 18 ans, chacun se verrait attribuer le premier niveau de qualification (et le salaire irrévocable qui va avec), et ensuite les institutions qui aujourd'hui qualifient les postes dans les conventions collectives ou certifient les personnes dans la validation des acquis de l'expérience auraient comme objet de qualifier des personnes et d'accompagner leurs progrès en la matière. Non pas de les certifier, c'est le rôle de l'école et cela atteste de la capacité à produire de la valeur d'usage : de les qualifier, c'est-à-dire de les inscrire dans la production de valeur économique et de leur attribuer un niveau de salaire, un salaire qui serait lié à leur

(7) Rappelons que le salaire à vie est lié à la qualification et non pas au statut : il peut concerner des travailleurs indépendants et permet des changements de statut très difficiles aujourd'hui. Il est déjà une réalité pour la fonction publique, mais dans une logique dominée.

(8) Cet article ne peut qu'évoquer brièvement un tel droit politique. Le lecteur en trouvera la présentation dans L'enjeu $d u$ salaire (FRIOT, 2012). Ce livre, par ailleurs, traite longuement d'une dimension décisive de la maîtrise de la valeur économique abordée en conclusion de cet article : le remplacement du crédit et de la propriété lucrative par la propriété d'usage et par le financement de l'investissement par une cotisation économique sur le modèle de la cotisation sociale. 
personne ${ }^{(9)}$. Confortées ainsi dans leur capacité à produire de la valeur économique, les personnes devraient postuler dans des collectifs de travail, et être estimées aptes à y demeurer, pour produire des valeurs d'usage. Un service civique pourvoirait aux tâches indispensables qui resteraient non réalisées.

Ce modèle du salaire tout au long de la vie rompt avec la modération salariale des vingt-cinq dernières années : continuer le salaire jusqu'à la mort sans réduction à l'occasion de la retraite, le continuer entre deux emplois, inclure dans le salaire l'ancienneté de la carrière, tout cela suppose la restauration du poids des salaires dans le PIB, qui a chuté depuis les années 1980. Ce serait aussi un salaire totalement mutualisé, puisqu'une telle «sécurité sociale professionnelle» ${ }^{(10)}$ repose sur la poursuite de la logique de sécurité sociale. C'est parce que ce n'est pas chaque employeur qui paye les frais de santé de ses salariés que la couverture santé est assurée, tout comme les allocations familiales ou les pensions ${ }^{(11)}$. La sécurité sociale nous sort de la logique du pollueur payeur, celle précisément que les réformateurs veulent développer. Étendre la mutualisation du salaire aux salaires directs eux-mêmes est la condition de leur distribution assurée à chacun, quels que soient les aléas de l'entreprise dans laquelle il travaille. Une cotisation salaires, prélevée elle aussi sur la valeur ajoutée au nom du droit du salaire socialisé comme le sont déjà les cotisations sociales, financerait les salaires à vie.

Ce salaire à vie à la qualification fait que l'horizon des individus s'élargit et se libère du chantage à l'emploi. La logique de l'emploi inscrit les employés dans leur statut de mineur social : tous leurs droits reposent sur la codification de leur subordination. $\mathrm{Au}$ contraire, les salariés ainsi titulaires d'une qualification ne seraient plus des demandeurs d'emploi, ils seraient posés comme producteurs, les seuls producteurs : ce ne seraient plus des mineurs sociaux. Le travail serait libéré de la subordination à un employeur, ce qui ne veut pas dire que ce serait un travail sans contrainte, sans échéance, sans hiérarchie, toutes choses qui sont inhérentes à la production. Mais la hiérarchie peut être élective par exemple; la contrainte peut relever de processus démocratiques de délibération entre des égaux également titulaires de qualifications certes inégales et progressant à des rythmes différents, mais dont l'amélioration est assurée par l'intervention des caisses de salaires et des

(9) Sur cette distinction fondamentale entre activité/valeur d'usage/certification d'une part et d'autre part travail/valeur économique/qualification, voir FrIOT, 2012.

(10) Pour reprendre l'expression de la CGT et l'opposer à la «sécurisation des parcours professionnels» propre à la dynamique du marché du travail et de l'employabilité.

(11) A contrario, la non mutualisation interprofessionnelle de la couverture des accidents du travail et maladies professionnelles interdit tout progrès significatif dans leur reconnaissance. institutions de la qualification. Par ailleurs, à partir du moment où les personnes seraient posées comme sujets de la création de valeur économique, ce qui leur est aujourd'hui rigoureusement refusé puisque cette qualité de sujet n'est reconnue qu'aux actionnaires, aux prêteurs et aux employeurs, l'économie deviendrait une affaire de «nous» et non de «ils». De même que le suffrage universel a suscité chez ses titulaires une responsabilité dans le champ politique, de même la qualification personnelle susciterait une responsabilité en matière de production de valeur économique, qui d'ailleurs revitaliserait la politique, aujourd'hui en pleine confusion du fait de l'impuissance collective face aux menées d'une petite minorité de sujets économiques.

Qu'en est-il de l'entreprise? Nous avons vu que dans la logique de l'emploi, l'entreprise est un espace distinct avec un périmètre bien défini alors que dans la logique du marché du travail l'entreprise est une réalité beaucoup plus fluctuante au gré des logiques de la création de valeur pour les actionnaires. Autant la logique de l'emploi affirmait sous un mode hétéronome l'existence d'un collectif de travail avec des collègues définis par l'employeur, autant la logique du marché du travail dévaste ces collectifs liés à l'employeur : les logiques de filialisation, de sous-traitance voire de prêt de maind'œuvre ont des effets relativement destructeurs sur les collectifs de travail, avec des effets en retour de personnalisation des réseaux de collègues, en particulier chez les cadres au fur et à mesure que le constat de l'incompatibilité entre la logique de l'actionnaire et celle de la production les pousse à une forme de cynisme vis-à-vis de l'entreprise. Ces désillusions de la financiarisation conduisent à des phénomènes d'individualisation des réseaux, des collectifs de travail. La question est d'inverser la logique actuelle, d'universaliser cet attribut personnel des collectifs de travail et de résoudre sur chaque lieu de travail les synergies entre ces qualifiés porteurs de réseaux de sorte qu'ils s'assument comme collègues dans des entreprises définies non par leur propriétaire mais par leur produit. Toutes les formes locales d'expérimentation qui se multiplient aujourd'hui dans des cadres coopératifs ou associatifs, voire de la société anonyme, seraient confortées à un niveau macro-économique par la maîtrise collective de la valeur économique.

* $\quad *$

La promotion de l'employabilité n'est pas l'unique issue possible de la mise en cause de l'emploi par les réformateurs : une nouvelle logique salariale peut au contraire trouver l'occasion de s'affirmer dans le recul de l'emploi. Toutes les thématiques de la dynamique de la qualification personnelle que je viens d'évoquer sont d'ailleurs 
déjà présentes, même si c'est en creux, dans les conflits et les négociations d'entreprise, de branche ou interprofessionnels. Mais il est vrai que leur crédibilité pâtit de deux phénomènes. D'une part la revendication du plein-emploi (quitte à le qualifier de «vrai», de «solidaire», etc.) reste l'horizon de l'action syndicale, alors que la revendication de la pleine qualification, c'est-à-dire de l'attribution à chacun d'une qualification, que rend possible le recul de l'emploi, est incompatible avec celle du plein-emploi. Revendiquer le retour de vrais employeurs en mesure de négocier la réinscription des droits salariaux dans l'emploi est à la fois une illusion et un recul, alors qu'avec la qualification personnelle, nous avons un support des droits salariaux en mesure de se substituer à l'emploi. D'autre part, le projet de salaire à la qualification personnelle n'est pas accompagné de propositions macro-économiques alternatives en matière de financement de l'investissement et de création monétaire.

Les possibles existent déjà pourtant et je conclus en ouvrant la réflexion sur des questions qui renvoient aux conditions de l'affirmation de la logique salariale. S'agissant de l'accumulation financière, du crédit bancaire, du prêt à intérêt, de la bourse, toutes ces institutions peuvent être remplacées en transposant pour le financement de l'investissement l'expérience de la cotisation sociale. Sur son modèle, on peut parfaitement imaginer une cotisation économique. L'investissement correspond à des engagements à bien plus court terme que les pensions. Et s'il est possible de financer les pensions au plus grand bénéfice des régimes et des pensionnés sans aucune accumulation financière, sans aucune logique d'épargne et de prêts, il est possible de financer l'investissement de la même façon en affectant une cotisation économique au salaire socialisé, prélevée sur la valeur ajoutée comme les cotisations sociales ou le salaire direct. Cette cotisation serait collectée par des caisses d'investissement qui financeraient sans taux d'intérêt ni remboursement, puisqu'il n'y aurait pas d'accumulation privée du capital, après délibération politique selon des procédures que nous pratiquons déjà à propos des investissements publics. On ne peut pas espérer sortir de la crise actuelle par le haut si on maintient la propriété lucrative, le crédit bancaire, les investisseurs et le retour sur investissement. Leur régulation nous conduira à une crise encore plus grave dans quelques années : c'est ce que nous observons depuis 30 ans. Avec la cotisation sociale, le salaire nous offre une expérience déjà longue du bénéfice que l'on tire à les supprimer. Quant à la création monétaire, tant que ce sont les anticipations par les banques commerciales sur le prix des marchandises capitalistes qui la fondent, il n'y aura pas de légitimité pour le travail non marchand, ou non subordonné, qui fait en permanence l'objet d'une suspicion d'être un parasite vivant des «prélèvements obligatoires». La création monétaire est toujours affaire d'anticipation de ce qui va être produit. Plutôt que d'anticiper le produit du travail et pire encore son seul produit marchand pour créer de la monnaie, pourquoi ne pas anticiper la capacité des individus à créer de la valeur économique telle qu'elle est mesurée par la qualification? La mesure de la production nouvelle estimée lors de la création monétaire ne serait plus la marchandise capitaliste, mais la qualification des salariés. Et l'instance ayant en charge la création monétaire agirait de concert avec l'instance gestionnaire des qualifications.

Ces propositions prêtent certes à débat, encore faut-il l'ouvrir. Épier tout ce qui, dans le réel, est porteur d'émancipation devrait être le cœur de la démarche du chercheur en sciences sociales. J'ai tenté de montrer comment la cotisation sociale ou la qualification des postes, initiatives au départ patronales, devenues ensuite insupportables au patronat qui a entrepris depuis de les remplacer par la prévoyance et l'employabilité, peuvent porter la sortie du marché du travail dans un salaire à la qualification à vie. En récusant le misérabilisme aujourd'hui dominant en sociologie du travail sur l'analyse des réformes, le présent texte souhaite contribuer à soutenir une émancipation déjà là. 


\section{Bibliographie}

Bozio A., Piketty T. (2008), Retraites : pour un système de comptes individuels de cotisations. Propositions pour une refonte générale des régimes de retraite en France, Éd. Rue d'Ulm.

Cahuc P., Kramarz F. (2004), De la précarité à la mobilité : vers une sécurité sociale professionnelle, Rapport au ministre de l'Économie et de l'Emploi, La Documentation française, novembre.

Castel N. (2009), La retraite des syndicats, Paris, La Dispute.

CAStel R. (1995), Les métamorphoses de la question sociale : une chronique du salariat, Paris, Fayard.

FRIOT B. (2007), «La proposition CGT de sécurité sociale professionnelle : de la sécurité sociale à la mise en cause du marché du travail», in Batifoulier Ph. et al. (dir.), Approches institutionnalistes des inégalités en économie sociale, tome I : les évolutions, Paris, L'Harmattan, pp. 361-375.

FRIOT B. (2009), «La revendication d'un statut du travail salarié à la CGT, enjeu d'unification du salariat?» in Bouffartigue P. et Béroud S. (dir.), Quand le travail se précarise, quelles résistances collectives? Paris, La Dispute, pp. 315-331.

Friot B. (2010), L'enjeu des retraites, Paris, La Dispute.

Friot B. (2012), Puissances du salariat, Paris, La Dispute (nouvelle édition augmentée à paraître, $1^{\text {re }}$ éd. 1998).
Friot B. (2012), L'enjeu du salaire, Paris, La Dispute.

Gobin C. (2005), «L’Union européenne : où est passé l'acteur?» Cahiers lillois d'économie et de sociologie, Université Lille 1, Paris, L'Harmattan, pp. 65-88.

Gobin C. (2005), «La transformation du social au cœur de la politique publique de l'Union européenne», Revue de l'Institut de Sociologie, Université Libre de Bruxelles, no 1-2, pp. 39-56.

LE LANN Y. (2006), La cohérence du projet Turner de réforme des retraites : individualisation et financiarisation de la pension britannique, Mémoire de M2, Paris X Nanterre.

Le LanN Y. (2009), «Le modèle suédois des retraites : le cheval de Troie de la patrimonialisation», Les Notes de l'IES, $\mathrm{n}^{\circ} 6$, août-septembre.

Ramaux C. (2006), Emploi : éloge de la stabilité. L'État social contre la flexicurité, Paris, Mille et une nuits.

Salais R. (2005), «Décrire et évaluer la pluralité des modèles sociaux en Europe : une approche en termes de dispositifs d'action publique», in Chatel E., Kirat T. et Salais R. (dir.), 2005, L'action publique et ses dispositifs : institutions, économie, politique, Paris, L'Harmattan, pp. $163-188$.

Supiot A. (dir.) (1999), Au-delà de l'emploi, Paris, Flammarion. 